\title{
Editorial: Misguided Macrophage and Risk of Coronary Atherosclerosis
}

\section{Afaque Alam $\mathbf{M d}^{*}$}

Department of Pediatrics, Drexel University College of Medicine, Philadelphia, USA

It has been well established that lipid laden macrophages critically involved in all stages of atherosclerosis, from initiation of abrasion, plaque development, to necrosis and plaque rupture to myocardial infarction. In this complex phenomenon, numerous inflammatory cytokines, chemokines, signaling molecules, proteins, small RNAs, transcription factors, and a plethora of receptors are involved.

But why there are resident macrophages in the plaque in the first place, and what do they do in the atherosclerotic lesion? The resident macrophages are multifaceted and possibly alter their forms and functions during the different stages of atherosclerosis. Typically macrophages are motile phagocytic cells and eat up nonself substances such as modified LDL through macropinocytosis or scavenger receptor-mediated pathways, resulting in the formation of the foam cells- a heavily lipid loaded, inactive, dormant macrophage - that are the manifestation of the atherosclerotic plaque. These lipid laden foam cells activates and secretes numerous receptors and pro-inflammatory markers (IL-1, IL-6, TNF, CCL2, CCL5 and CXCR4) makes the lesion more vulnerable [1]. It also secretes macrophage retention factors such as netrin 1 and semaphorin 3E, which further block their escape route [2]. The magnitude of lipid and cholesterol loading, activation of receptors, and metabolic state of the cells, make them misguided and immotile.

Nevertheless these resident macrophages become misguided and trapped in the lesion. The consequences to this "cluelessness" are tremendous. In the accompanying article, Dr. Kathryn Moore and colleagues [2] describe elegantly experimental evidence that helps us understand why macrophages become clueless to get out from the lesion. Molecules such as netrin-1 appear to be key players in this phenomenon.

It helps to review first briefly netrin-1 and its role in resident macrophages (Figure 1). Netrins are secreted proteins and structurally resembles the extracellular matrix protein laminin. Firstly, it has been discovered as guidance cues for cell and axonal migration in the central nervous system [3]. It mediates axonal chemoattractant activity via binding to the deleted in colorectal cancer (DCC) and neogenin receptors, and repulsion via the uncoordinated-5 (UNC5) receptors $[3,4]$. Over the years it has been established that netrins can play a vital role in multiple organs and tissues, including the vasculature, heart, lung, pancreas, muscle and mammary gland, by mediating cell migration, cell-cell interactions and cell-extracellular matrix adhesion. Of note, the role of netrin-1 in atherosclerosis and retention of macrophages in the plaque is highly debatable.

Accumulating data have established the signal cues for netrin- 1 and its receptors outside the nervous system, for example in atherosclerosis [2], organogenesis [5], angiogenesis [6] and tumorigenesis [7], proposing that netrin-1 modulates cell migration in a broader context. It has also been found to be upregulated in many diseases [8-10], and can easily be measured in blood plasma. Thus it would be good biomarkers for the risk and severity assessment of the disease.

Controversy exists regarding the identity of endothelial receptors mediating these effects in the vasculature. Several others have reported that the inhibition of netrin-1 canonical receptors, DCC, neogenin, and the Unc5b $[5,10]$ did not abrogate netrin signaling [11] and additional findings have reinforced the idea that a one-to-one relationship between ligand and receptor is too simplistic.

Why macrophages become clueless to get out from the lesion? The mechanism by which lipid-laden macrophage gets trapped is poorly understood. It could be speculate that some proteins or signal may obstruct with macrophage migratory signals which may prolong the stay of macrophages in the lesion. However, of late, van gill and colleagues published remarkable findings why these misguided macrophages trapped in atherosclerotic lesion in Nature Immunology. They found that signal blocker protein called netrin1, known for the central nervous system. Authors have suggested that netrin 1 was also secreted by resident macrophages activated by oxLDL in the plaque of atherosclerotic mice, where it inactivates the migration of lipid laden resident macrophages in response to chemical cues (CCR7) linked to their egress from plaques, hence these cells accumulated within the plaque. However, in another experiment in netrin-1 knockout mice they observed reduced atherosclerotic symptoms and resulted in fewer plaque-resident macrophages [2]. Findings of the research suggested that netrin-1 proteins are involved in multiple physiological responses, ranging from angiogenesis to inflammation, making it an attractive potential therapeutic target. On the other hand, studies evaluating the potential clinical benefits of up- and down-regulation of netrin-1 have yielded seemingly contradictory results $[2,12]$.

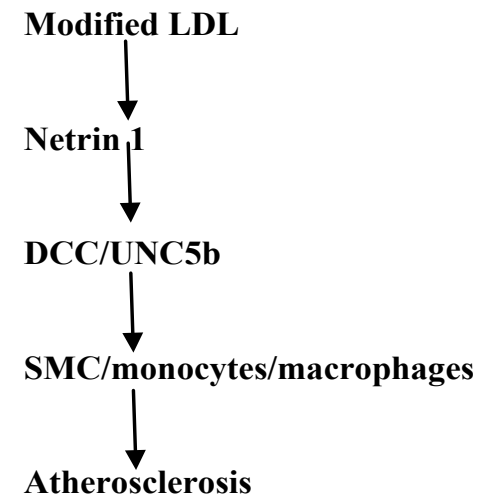

Figure 1: Brief review of netrin-1 and its role in resident macrophages.

*Corresponding author: Afaque Alam Md, Department of Pediatrics, Drexel University College of Medicine, Philadelphia, PA 19102, USA, Tel: +1 (901) 5186443; E-mail: aafaqq@gmail.com

Received June 02, 2016; Accepted June 03, 2016; Published June 10, 2016

Citation: Alam MA (2016) Editorial: Misguided Macrophage and Risk of Coronary Atherosclerosis. J Vasc Med Surg 4: e118. doi:10.4172/2329-6925.1000e118

Copyright: ( 2016 Alam MA. This is an open-access article distributed under the terms of the Creative Commons Attribution License, which permits unrestricted use, distribution, and reproduction in any medium, provided the original author and source are credited. 
Citation: Alam MA (2016) Editorial: Misguided Macrophage and Risk of Coronary Atherosclerosis. J Vasc Med Surg 4: e118. doi:10.4172/23296925.1000 e118

Page 2 of 2

With the increasing understanding of the role of netrin1, oxLDL and resident macrophages, new research areas and treatment strategies are beginning to emerge. Based on these data, future research in this area could benefit in identifying potential diagnostic biomarkers and novel therapies for coronary heart disease.

\section{References}

1. Chistiakov DA, Bobryshev YV, Orekhov AN (2016) Macrophage-mediated cholesterol handling in atherosclerosis. J Cell Mol Med 20: 17-28.

2. van Gils JM, Derby MC, Fernandes LR, Ramkhelawon B, Ray TD, et al. (2012) The neuroimmune guidance cue netrin-1 promotes atherosclerosis by inhibiting the emigration of macrophages from plaques. Nat Immunol 13: 136-143.

3. Laumonnerie C, Da Silva RV, Kania A, Wilson SI (2014) Netrin 1 and Dcc signalling are required for confinement of central axons within the central nervous system. Development 141: 594-603.

4. Grandin M, Meier M, Delcros JG, Nikodemus D, Reuten R, et al. (2016) Structural Decoding of the Netrin-1/UNC5 Interaction and its Therapeutical Implications in Cancers. Cancer Cell 29: 173-185.

5. Dalvin S, Anselmo MA, Prodhan P, Komatsuzaki K, Schnitzer JJ, et al.
(2003) Expression of Netrin-1 and its two receptors DCC and UNC5H2 in the developing mouse lung. Gene Expr Patterns 3: 279-283.

6. Ahmed RP, Haider KH, Shujia J, Afzal MR, Ashraf M (2010) Sonic Hedgehog gene delivery to the rodent heart promotes angiogenesis via iNOS/netrin-1/ PKC pathway. PLoS One 5: e8576.

7. Arakawa $\mathrm{H}$ (2004) Netrin-1 and its receptors in tumorigenesis. Nat Rev Cancer 4: $978-987$.

8. Nikolopoulos SN, Giancotti FG (2005) Netrin-integrin signaling in epithelial morphogenesis, axon guidance and vascular patterning. Cell Cycle 4: e131135.

9. Joseph BB, Quan PD (2013) The neuroimmune guidance cue netrin-1: a new therapeutic target in cardiovascular disease. Am J Cardiovasc Dis 3: 129-134.

10. Ramesh G, Berg A, Jayakumar C (2011) Plasma netrin-1 is a diagnostic biomarker of human cancers. Biomarkers 16: 172-180.

11. Yebra M, Montgomery AM, Diaferia GR (2003) Recognition of the neural chemoattractant netrin-1 by integrins alpha6beta4 and alpha3beta1 regulates epithelial cell adhesion and migration. Dev Cell 5: 695-707.

12. Khan JA, Cao M, Kang BY, Liu Y, Mehta JL, et al. (2011) Systemic human Netrin-1 gene delivery by adeno-associated virus type 8 alters leukocyte accumulation and atherogenesis in vivo. Gene Ther 18: 437-444. 